\title{
Indicadores de suicidio: comparación entre feminicidas y maltratadores.
}

Suicidal markers: a comparison between femicide perpetrators and batterers.

\author{
Laura Blanco Iglesias ${ }^{1}$ \\ Jorge Santos Hermoso ${ }^{1}$ \\ Manuel de Juan Espinosa ${ }^{2}$ \\ José Luis González Álvarez ${ }^{3}$
}

(1) Universidad Autónoma de Madrid, Madrid, España.

(2) Instituto de Ciencias Forenses y de la Seguridad. Universidad Autónoma de Madrid, Madrid, España.

(3) Secretaría de Estado de Seguridad, Ministerio del Interior, Madrid, España.

Email correspondencia:. lau.blancoiglesias@gmail.com

\section{Resumen}

La literatura actual y los instrumentos de valoración del riesgo existentes consideran las conductas suicidas previas del autor de feminicidio como un factor de riesgo importante. El presente estudio tiene como objetivo identificar si los indicadores de suicidio previos en el agresor pueden ser factores de riesgo de feminicidio y de reincidencia en maltratadores en España. Para ello, se comparó una muestra de 150 casos de feminicidio del estudio de Revisión Pormenorizada de Homicidio de Violencia de Género y 450 casos control de maltratadores extraídos aleatoriamente del sistema VioGén. Los resultados indican que la presencia de dichos indicadores era más probable en feminicidas que en maltratadores, incluso si ambos tenian una historia de violencia previa; más probable en maltratadores reincidentes que en no reincidentes; y más probable en feminicidas con antecedentes de violencia previa que en aquellos que no tenian. Comparando maltratadores reincidentes y no reincidentes por un lado, y feminicidas con y sin violencia previa por otro, no se observaron diferencias en intentos o ideas de suicidio. Cuando se compararon feminicidas con violencia previa y maltratadores reincidentes, no se observaron diferencias en las amenazas de suicidio. Estos resultados pueden ayudar a las Fuerzas y Cuerpos de Seguridad a prevenir el desenlace mortal y la reincidencia del autor, y por otro lado permiten el ajuste de los programas de intervención con agresores y víctimas de violencia de género.

Palabras Clave: Violencia de género, feminicidio, indicadores de suicidio, factor de riesgo, Sistema VioGén.

\begin{abstract}
The current literature and the existent risk assessment tools consider prior suicidal behaviour of the femicide perpetrator to be an important risk factor. The aim of this study is to identify to which extent previous suicidal markers in the perpetrator may be risk factors for femicide and recidivism in batterers in Spain. To that aim, 150 femicide cases from the Domestic Homicide Review study and 450 batterer control cases randomly chosen from the VioGén system were compared. Results show that suicidal markers were more likely to take place in femicide perpetrators than in batterers, even if they had a record of previous violence; more likely in reoffending batterers than in non-reoffending batterers; and more likely in femicide perpetrators with a record of violence than those with no record. No differences were found in the suicide attempt variable for the reoffending batterers and non-reoffending batterers comparison, nor for the femicide perpetrators with previous violence records and those with no records comparison. No differences were found either regarding suicide threats in the non-reoffending batterers and femicide perpetrators with no records of previous violence comparison. These results could help Law Enforcement Agencies to prevent deadly outcomes and the perpetrator's recidivism. Additionally, these results enable experts to adjust intervention programmes for perpetrators and victims of domestic violence.
\end{abstract}

Keywords: Domestic violence, femicide, suicidal markers, risk factor, VioGén System.

Fecha de envío: 02/10/2019

Fecha de aceptación: 01/12/2019 


\section{INTRODUCCIÓN}

El homicidio seguido de suicidio (H-S) es un fenómeno especialmente vinculado a los crímenes que se dan en el ámbito familiar, principalmente al homicidio de pareja o feminicidio (González, Sánchez, López-Ossorio, Santos \& Cereceda, 2018; López-Ossorio et al., 2018; Vatnar, Friestad \& Bjørkly, 2019). Del total de los autores de feminicidio, aproximadamente el 34\% se suicidan después de cometer el homicidio (López-Ossorio et al., 2018). Esta prevalencia supera con creces la tasa de suicidio que se encuentra en otros tipos de homicidios (Barber at al., 2008; Campbell, Glass, Sharps, Laughon, \& Bloom, 2007; Comstock et al., 2005), así como la prevalencia en la población general (Bridger, Strang, Parkinson \& Sherman, 2017). En base a estos datos, cabe preguntarse si las conductas suicidas previas al delito de los feminicidas servirían como indicadores de riesgo letal, por estar más presentes en feminicidas que en aquellos agresores que ejercen violencia no letal contra su pareja.

La literatura internacional encuentra una relación significativa entre la Violencia Contra la Pareja (VCP) física, grave y letal y los comportamientos suicidas del agresor previos al hecho (ideación suicida e intentos de suicidio), no siendo así en el caso de violencia doméstica física leve (Echeburúa, Fernández-Montalvo \& Corral, 2008) o en otros delitos violentos (Belfrage y Rying, 2004; Bridger et al., 2017; Button, Angel \& Sherman, 2017; Chalkley \& Strang, 2017; Liem, Hengeveld \& Koenraadt, 2009; Thornton, 2017). Bridger et al. (2017) encuentran que una de las variables que más predice el crimen es la ideación suicida. Otras variables, como el consumo de alcohol crónico y la convivencia con la víctima, también predicen el feminicidio; no obstante, estas son muy prevalentes en la población general, por lo que los autores advierten que fijar la atención en estas variables podría conllevar una predicción desproporcionada e incorrecta del feminicidio. Ocurre lo contrario con la ideación suicida: su prevalencia en la población es más baja que en muestras de otros tipos de homicidios, por lo que podría ser un predictor adecuado. No obstante, entre estos estudios internacionales, pocos cuentan con grupo control, y, aquellos que lo tienen, su grupo no es adecuado para extraer conclusiones válidas (Bridger et al., 2017; Button, Angel \& Sherman, 2017; Chalkley \& Strang, 2017; Thornton, 2017).

En España, Echeburúa et al. (2008) compararon una muestra de casos de VCP de violencia grave y una muestra de casos de VCP no tan grave, con el objetivo de establecer qué diferenciaba a víctimas y agresores en cada tipo de violencia, encontrando variables que diferenciaban entre unos agresores y otros. Los agresores letales suelen tener una nacionalidad distinta a la española, y es más probable que ejerzan la violencia cuando la pareja se está separando, cuando hay conductas de acoso durante las semanas previas a la agresión, y cuando hay hijos de anteriores parejas de por medio. Además, presentar dificultades económicas, carecer de una red social adecuada de apoyo, quebrantar órdenes de alejamiento y consumir sustancias supone también un mayor riesgo de que el agresor ejerza violencia letal y grave. En sus resultados destacan también que, tanto las amenazas de suicidio como los intentos, se daban de forma más significativa en agresores que ejercían violencia grave. Aparte de este resultado puntual, que se sepa en la literatura española no se ha replicado ningún estudio extranjero sobre los indicadores de suicidio como factor de riesgo de feminicidio.

A este respecto, a nivel internacional las herramientas existentes de evaluación de la probabilidad de letalidad en casos de VCP incluyen las amenazas y los intentos de suicidio del agresor como indicadores de riesgo, como es el caso del instrumento Danger Assesment (Campbell, 2004; Campbell, Webster \& Glass, 2009). Por otro lado, también se han desarrollado herramientas de predicción del riesgo de reincidencia en el ámbito de la violencia de género, como el protocolo SARA (Kropp \& Hart, 2000) y el DASH (Richards, 2009), incluyendo ambos la conducta suicida previa del agresor. Concretamente, se incluyen amenazas de suicidio en el caso del DASH, ideación suicida en el SARA, e intentos de suicidio en ambos. En España se implementaron protocolos de valoración policial del riesgo de reincidencia en 2007, con la puesta en marcha del Sistema de Seguimiento Integral en los casos de Violencia de Género (Sistema VioGén; González, LópezOssorio \& Muñoz, 2018; de Juan, González, Liberatore, Quijano \& Torrecilla, 2017). El sistema VioGén integra en una sola plataforma web el seguimiento y la coordinación de los asuntos de violencia de género, y acceden a ella miembros de las Fuerzas y Cuerpos de Seguridad, de Instituciones Penitenciarias, Juzgados, Fiscalías, Institutos de Medicina Legal y Forense, etc.

El Protocolo de las Fuerzas y Cuerpos de Seguridad 
(FCS) en VioGén incluye un primer formulario de Valoración Policial de Riesgo (VPR), y otro posterior de Valoración Policial de la Evolución de Riesgo (VPER). El VPR se cumplimenta cuando se presenta una denuncia por violencia de género, y ofrece una valoración integral del riesgo que corre la víctima de volver a sufrir violencia por parte del agresor. El VPER, por otro lado, se pone en marcha cuando el autor ha sido denunciado previamente, con el objetivo de hacer un seguimiento de la evolución del riesgo del caso. Tanto el VPR como el VPER tienen en cuenta variables de víctima y agresor, y ofrecen una valoración del riesgo que puede oscilar entre los niveles "no apreciado" "bajo", "medio", "alto" y "extremo" (González, López-Ossorio et al., 2018).

Ambas herramientas predictivas cuentan con tres indicadores de riesgo de reincidencia para la VCP que tienen que ver con la tendencia suicida del agresor: intentos de suicidio previos, ideación suicida y amenazas de suicidio (López-Ossorio, González-Álvarez \& AndrésPueyo, 2016); resultando que dichos indicadores presentaban una asociación estadísticamente significativa con la reincidencia policial en VCP en los siguientes seis meses (López-Ossorio, González, Buquerín, García \& Buela-Casal, 2017). Los dos formularios han sido validados $\mathrm{y}$ mejorados en versiones sucesivas para asegurar una precisión cada vez mayor (de Juan et al., 2017; LópezOssorio, González-Álvarez, Muñoz-Vicente, Urruela \& Andrés-Pueyo, 2019; López-Ossorio, Loinaz \& GonzálezÁlvarez, 2019).

El objetivo principal del presente estudio es comprobar si, tal y como sugieren los hallazgos internacionales (principalmente los más recientes de Chalkley \& Strang, 2017, y Thornton, 2017, en el Reino Unido), las conductas de suicidio previas del agresor, incorporadas en la valoración policial del riesgo de reincidencia en España, podrían servir también como factores de riesgo de feminicidio. En caso afirmativo, permitirá que los agentes de las FCS especializados en la valoración policial del riesgo pudieran prevenir el desenlace fatal. Para ello, en este estudio se compararán feminicidas (casos) con maltratadores (controles).

Por otro lado, como objetivo secundario, también se va a estudiar el impacto de los indicadores de suicidio sobre la reincidencia, tanto en la muestra de maltratadores como en la de feminicidas. Respecto a esta última, se considerará reincidentes a los homicidas que contaban con al menos una denuncia previa por violencia de género, o bien con motivos fehacientes para haber sido denunciados. Con esto se pretende proporcionar una variable adecuada para la predicción y prevención eficaz de la reincidencia en el ámbito de la VCP al personal de Instituciones Penitenciarias y de la Administración de Justicia.

Las hipótesis, formuladas en base a la literatura científica revisada, son las siguientes:

Hipótesis 1. La presencia de indicadores de suicidio estará más asociada a los feminicidas (es decir, aquellos agresores que ejercieron violencia letal contra su pareja o expareja) que a los maltratadores (aquellos que ejercieron violencia no letal) (Chalkley \& Strang, 2017; Echeburúa et al., 2008; Thornton, 2017).

Hipótesis 2. La presencia de indicadores de suicidio estará más asociada a los maltratadores reincidentes que a los no reincidentes (de Juan et al., 2017; González, López-Ossorio et al., 2018; López-Ossorio et al., 2016).

Hipótesis 3. La presencia de indicadores de suicidio diferirá de forma significativa entre los feminicidas con antecedentes de violencia previa en el ámbito de la violencia de género respecto a aquellos que no tienen antecedentes, dado que la violencia previa en la pareja se considera un factor de riesgo para la reincidencia, al igual que los indicadores de suicidio (Campbell et al., 2009; Kropp \& Hart, 2000; López-Ossorio et al., 2016; López-Ossorio et al., 2017; Richards, 2009). Concretamente, se hipotetiza que los feminicidas con antecedentes de violencia previa tendrán una mayor presencia de indicadores de suicidio comparados con aquellos que no tienen antecedentes, dado que esta violencia previa se podría considerar similar a la que ejercen los maltratadores reincidentes.

Hipótesis 4. La presencia de indicadores de suicidio estará más asociada a autores de feminicidio con antecedentes de violencia previa que a maltratadores reincidentes, ya que dichos indicadores suelen aparecer asociados a autores de feminicidio en mayor medida que a maltratadores (Chalkley \& Strang, 2017; Echeburúa et al., 2008; Thornton, 2017).

\section{MÉTODO}

\section{Muestra}

La muestra la componen 600 casos, de los cuales 150 son feminicidios que se obtuvieron del estudio nacional 
desarrollado por el EHVdG (González, Garrido et al., 2018), y 450 son casos de violencia de género no mortal extraídos aleatoriamente del Sistema VioGén de entre los que se dieron de alta entre octubre y diciembre de 2016 (inclusive).

La media de edad de los feminicidas fue de 45.8 años $(M=45 ; D T=14.53$; rango $=19-86)$, y en el caso de los maltratadores fue de 37.4 años $(M=36 ; D T=12.66$; rango $=14-81)$. El 70.7\% de los feminicidas eran españoles, y el $85 \%$ de los agresores no letales.

\section{Procedimiento}

Para el presente estudio se emplea un diseño ex post facto de casos (feminicidios) y controles (maltratadores no homicidas) intersujeto. Teniendo en cuenta la recomendación de Pardo y San Martín (2015), se seleccionaron tres casos de grupo control por cada caso.

Como variables dependientes se crearon tres. La variable tipo de agresor, con dos categorías $(1=$ "Maltratador" y 2 = "Feminicida"). En el caso de los maltratadores, se creó la variable reincidente $(1=$ "MR" y 2 = "MNR"), tomando como criterio que tuviera VPER con incidencia registrado en el Sistema VioGén. En el caso de los feminicidas, se creó la variable historial de violencia $(1=$ "FHV" у 2 = "FSHV"), a partir de la información recopilada por el EHVdG, incluyéndose entre los feminicidas con historial de violencia aquellos con denuncia registrada, pero también aquellos casos en los que se encontraron evidencias de violencia en la relación por parte del hombre, aun sin que esta fuera denunciada.

Las variables independientes fueron tres, relacionadas con el comportamiento suicida. Eligiéndose la existencia de amenazas de suicidio, variable obtenida del indicador 7 del formulario VPR, en la que se hace referencia a las amenazas de suicidio que profiere el agresor contra su pareja o expareja; y los intentos previos y la ideación suicida, indicador 21 del VPR, que incluye tanto la existencia de intentos previos de suicidio, como las manifestaciones recurrentes del agresor de acabar con su vida. Por último, se creó la variable indicadores comportamentales de suicidio (ICS), en la que se recogió la presencia de al menos una de las anteriores variables en el agresor. Es decir, que, si bien las dos primeras variables hacían referencia a comportamientos concretos, en la variable ICS se recoge la presencia de una o ambas variables. Todas las variables relacionadas con el suicidio fueron codificadas de manera dicotómica (1 = "presencia" y 2 = "ausencia").
Como análisis estadísticos, primero se llevó a cabo un análisis descriptivo para conocer la distribución de las variables de análisis, y, posteriormente, para la comprobación de las hipótesis propuestas se empleó el test de independencia Ji-Cuadrado, calculándose la medida de asociación odds ratio para la estimación del riesgo, en los cruces significativos.

\section{RESULTADOS}

De los 450 casos de maltratadores, el $20 \%$ fueron reincidentes; mientras que, en el grupo de 150 feminicidas, en el $70.5 \%$ de los casos se identificó historial de violencia previa.

Se encontraron diferencias estadísticamente significativas entre el tipo de agresor y las tres variables de suicidio: presencia de amenazas de suicidio $(\mathrm{OR}=2.06$; $\mathrm{CI}$ = 1.19-3.57), intentos/ideación suicida $(\mathrm{OR}=2.89 ; \mathrm{CI}=$ 1.8-4.66), e ICS (OR = 3.06; CI = 1.97-4.74), asociándose las tres variables al grupo de feminicidas (Tabla 1).

Tabla 1. Comparación entre tipo de agresor y las variables de suicidio.

\begin{tabular}{|c|c|c|c|}
\hline \multirow{2}{*}{ Variable } & Maltratador & Feminicida & \multirow{2}{*}{$\chi^{2}$} \\
\hline & $\mathrm{n}(\%)$ & $\mathrm{n}(\%)$ & \\
\hline \multicolumn{3}{|c|}{ Amenazas suicidio } & $6.93 * *$ \\
\hline Sí & $38(8.4)$ & $24(16)$ & \\
\hline No & 412 (91.6) & $126(84)$ & \\
\hline \multicolumn{3}{|c|}{ Intentos/ideación suicida } & $20.19 * * *$ \\
\hline Sí & 45 (11.2) & $40(26.7)$ & \\
\hline No & $358(88.8)$ & $110(73.3)$ & \\
\hline \multicolumn{3}{|l|}{ ICS } & $26.56^{* * *}$ \\
\hline Sí & $60(13.3)$ & $48(32)$ & \\
\hline No & $390(86.7)$ & $102(68)$ & \\
\hline
\end{tabular}

$\mathrm{Al}$ analizar el grupo de maltratadores en función de la reincidencia (Tabla 2), la presencia de amenazas de suicidio $(\mathrm{OR}=2.26 ; \mathrm{CI}=1.11-4.62)$ y de los ICS $(\mathrm{OR}=1.9 ; \mathrm{CI}=$ 1.03-3.48) se asoció a maltratadores reincidentes, no encontrándose diferencias con las otras dos variables de suicidio.

Al comparar los feminicidas con y sin historial de violencia, tanto las amenazas de suicidio $(\mathrm{OR}=12.06$; $\mathrm{CI}$ $=1.57-92.37)$ como los ICS $(\mathrm{OR}=2.77$; CI = 1.17-6.55), se asocian a los FHV (Tabla 3).

Al comparar MR con el grupo de FHV (Tabla 4), se 
han encontrado diferencias significativas entre la presencia de intentos o ideas de suicidio del agresor $(\mathrm{OR}=2.45$; $\mathrm{CI}$ $=1.17-5.14)$, de ICS (OR = 2.46; CI = 1.29-4.71), siendo más comunes en los feminicidas. No se han encontrado diferencias en cuanto a las amenazas de suicidio.

Tabla 2. Comparación entre MR y MNR y las variables de suicidio.

\begin{tabular}{|c|c|c|c|}
\hline \multirow{2}{*}{ Variable } & MR & MNR & \multirow{2}{*}{$\chi^{2}$} \\
\hline & $\mathrm{n}(\%)$ & $\mathrm{n}(\%)$ & \\
\hline \multicolumn{3}{|c|}{ Amenazas suicidio } & $5.24 *$ \\
\hline Sí & $13(14.4)$ & $25(6.9)$ & \\
\hline No & 77 (85.6) & $335(93.1)$ & \\
\hline \multicolumn{3}{|c|}{ Intentos/ideación suicida } & 1.60 \\
\hline Sí & $12(15.2)$ & $33(10.2)$ & \\
\hline No & $67(84.8)$ & $291(89.8)$ & \\
\hline \multicolumn{3}{|l|}{ ICS } & $4.33^{*}$ \\
\hline Sí & $18(20)$ & $42(11.7)$ & \\
\hline No & $72(80)$ & $318(88.3)$ & \\
\hline
\end{tabular}

Tabla 3. Comparación entre FHV y FNHV y las variables de suicidio.

\begin{tabular}{|c|c|c|c|}
\hline \multirow{2}{*}{ Variable } & FHV & FSHV & \multirow{2}{*}{$\chi^{2}$} \\
\hline & $\mathrm{n}(\%)$ & $\mathrm{n}(\%)$ & \\
\hline \multicolumn{3}{|c|}{ Amenazas suicidio } & $8.84 * *$ \\
\hline Sí & $23(21.9)$ & $1(2.3)$ & \\
\hline No & $82(78.1)$ & $43(97.7)$ & \\
\hline \multicolumn{3}{|c|}{ Intentos/ideación suicida } & 2.39 \\
\hline Sí & $32(30.5)$ & $8(18.2)$ & \\
\hline No & $73(69.5)$ & $36(81.8)$ & \\
\hline \multicolumn{3}{|l|}{ ICS } & $5.63^{*}$ \\
\hline Sí & $40(38.1)$ & $8(18.2)$ & \\
\hline No & 65 (61.9) & $36(81.8)$ & \\
\hline
\end{tabular}

Tabla 4. Comparación entre MR y FHV y las variables de suicidio.

\begin{tabular}{|c|c|c|c|}
\hline \multirow{2}{*}{ Variable } & MR & FHV & \multirow{2}{*}{$\chi^{2}$} \\
\hline & $\mathrm{n}(\%)$ & $\mathrm{n}(\%)$ & \\
\hline \multicolumn{3}{|c|}{ Amenazas suicidio } & 1.79 \\
\hline Sí & $13(14.4)$ & $23(21.9)$ & \\
\hline No & 77 (85.6) & $82(78.1)$ & \\
\hline \multicolumn{3}{|c|}{ Intentos/ideación suicida } & $5.79 *$ \\
\hline Sí & $12(15.2)$ & $32(30.5)$ & \\
\hline No & $67(84.8)$ & $73(69.5)$ & \\
\hline \multicolumn{3}{|l|}{ ICS } & $7.59 * *$ \\
\hline Sí & $18(20)$ & $40(38.1)$ & \\
\hline No & $72(80)$ & $65(61.9)$ & \\
\hline
\end{tabular}

\section{DISCUSIÓN Y CONCLUSIONES}

Este estudio, que se sepa, es el primero en España que establece comparaciones directas entre la presencia de indicadores de suicidio previos en el agresor y su probabilidad de cometer un feminicidio o de reincidir en el ámbito de la violencia de género. Los resultados, en conjunto, muestran que la presencia de dichos indicadores es más probable en feminicidas que en maltratadores, incluso si ambos tenían una historia de violencia previa; más probable en maltratadores reincidentes que en no reincidentes; y más probable en feminicidas con antecedentes de violencia previa que en aquellos que no tenían.

Los resultados obtenidos para las tres variables (amenazas de suicidio, intentos o ideas de suicido e ICS) son congruentes con la primera de las hipótesis formuladas: es tres veces más probable que los feminicidas presenten dichos indicadores en relación con los maltratadores. Estos datos concuerdan con estudios previos que comparan autores de VCP extrema con autores de VCP más leve y no letal (Echeburúa et al., 2008). Por otro lado, la literatura que compara feminicidas con otros tipos de agresores también destaca para los feminicidas un mayor riesgo de poseer indicadores de suicidio previos al hecho (Belfrage y Rying, 2004; Bridger et al., 2017; Chalkley y Strang, 2017; Loinaz, Marzabal y Andrés-Pueyo, 2018; Thornton, 2017). Los indicadores de suicidio son, por tanto, un factor de riesgo de la comisión de un feminicidio en España. La razón por la cual se hayan presentes en mayor medida en este tipo de autores puede estar relacionada con el abuso de sustancias o la ruptura de la relación que mantenía con la víctima (Campbell et al., 2003; Echeburúa et al., 2008; Kivisto, 2015); sin embargo, esto es algo que todavía se debe estudiar en profundidad.

Los resultados también apoyan la segunda de las hipótesis (reincidencia), en la línea de la literatura científica previa (Kropp y Hart, 2000; López-Ossorio et al., 2016; López-Ossorio et al., 2017; Richards, 2009): en España parece ser dos veces más probable que los maltratadores reincidentes presenten amenazas de suicidio e ICS. Confirmando así lo idóneo de que los instrumentos de valoración del riesgo de reincidencia a nivel policial en España (VPR y VPER) incorporen las amenazas de suicidio como un factor de riesgo (López-Ossorio et al., 2016; López-Ossorio et al., 2017). No obstante, el resultado respecto a la variable de intentos e ideas de suicidio no apoya la hipótesis formulada. Esto puede deberse al posible carácter instrumental de las amenazas de suicidio. Los agresores reincidentes podrían emplear estas 
amenazas como método de control de la víctima, mientras que los no reincidentes, que solamente ejercieron violencia de forma puntual, no tendrían la necesidad de amenazar. Los intentos de suicidio, sin embargo, son más difíciles de llevar a cabo, más costosos que las amenazas, e implican un mayor peligro para el agresor. Es decir, no serían una herramienta útil y eficaz para ejercer control sobre la víctima. Por tanto, al no tener un carácter instrumental ni estar relacionados con la manipulación de la víctima, los intentos de suicidio no constituyen una variable diferenciadora entre agresores reincidentes y no reincidentes. Además, las amenazas de suicidio están ligadas a la víctima. Es decir, el agresor amenaza a otra persona con el objetivo de controlarla, manipularla, o de ejercer violencia psicológica contra ella; por esta razón, tiene sentido que las amenazas aparezcan asociadas a la reincidencia. En cambio, los intentos de suicidio suelen ser independientes de la víctima, ya que el objetivo es el propio agresor. Por tanto, al ser fenómenos de diferente intencionalidad y etiología, estarán mediados por diferentes variables, no controladas en este estudio, lo que también podría explicar la diferencia en los resultados.

Con respecto a la historia de violencia del grupo feminicida, los resultados obtenidos son congruentes con la tercera de las hipótesis en amenazas de suicidio y en ICS, pero no en los intentos o ideas de suicidio. Estos resultados no pueden compararse con otros estudios similares, ya que la literatura científica previa no ha comparado feminicidas que ejercieron violencia previa con aquellos que no para estas variables en concreto. Además, la mayor parte de los estudios que analizan la violencia se basan únicamente en datos de denuncias anteriores (Bridger et al., 2017; Chalkley y Strang, 2017; Thornton, 2017), dejando fuera del análisis muchos casos en los que el agresor no ha sido denunciado, pero sí había ejercido violencia (Eke et al., 2011). Estos resultados son similares a los de la comparación entre maltratadores reincidentes y no reincidentes, por lo que se podría extrapolar la explicación de la instrumentalidad de las amenazas de suicidio en el caso de los autores de feminicidio con antecedentes de violencia previa, ya que sería una forma de mantener controlada a la víctima a lo largo del tiempo. Los intentos de suicidio, por otro lado, no serían instrumentales, lo que explicaría que no se hayan encontrado diferencias significativas entre los autores que ejercieron violencia previa y entre los que no la ejercieron.

Los resultados apoyan la cuarta hipótesis de forma parcial, sobre reincidencia en ambos grupos. Tanto los intentos o ideas de suicidio como el ICS se presentan asociados a los autores de feminicidio que han ejercido violencia previa, no ocurriendo lo mismo con las amenazas de suicidio, un resultado que difiere de forma llamativa del de los dos análisis anteriores. El hecho de que las amenazas de suicidio no sean de utilidad para diferenciar entre maltratadores reincidentes y feminicidas que ejercieron violencia previa, aunque a primera vista pueda parecer contradictorio respecto a los análisis presentados previamente, no hace más que fortalecer la interpretación de los mismos. En este caso, ambos tipos de agresores buscan controlar a la víctima durante un período de tiempo, y para ello necesitan un método eficaz para que ésta permanezca a su lado. Es lógico que tanto feminicidas como maltratadores reincidentes empleen las amenazas de suicidio de forma instrumental con el mismo objetivo y que, entonces, estas no constituyan una variable útil para diferenciar entre ambos grupos.

En suma, ningún estudio español había examinado de forma tan específica la influencia de la reincidencia (o antecedentes de violencia previa en el caso de los feminicidas) en los indicadores de suicidio presentados por ambos tipos de agresores. No obstante, teniendo en cuenta lo que refleja la literatura sobre conducta suicida en feminicidas (Bridger et al., 2017; Button et al., 2017; Chalkley \& Strang, 2017; Echeburúa et al., 2008; Thornton, 2017) y en agresores reincidentes (de Juan et al., 2017; Kropp \& Hart, 2000; López-Ossorio et al., 2016; López-Ossorio et al., 2017; Richards, 2009), los resultados de este trabajo apuntan en la misma línea. Además, confirman que, a pesar de que ambos tipos de agresores tengan una historia de violencia previa, los intentos o ideas de suicido siguen constituyendo un factor de riesgo de feminicidio.

La diferencia en los intentos o ideas de suicidio puede deberse a las propias características del autor feminicida. La literatura ha demostrado que estos agresores suelen tener una serie de problemas personales específicos (abuso de sustancias, desempleo, la ruptura de la relación de pareja, etc.) que pueden explicar que la presencia de esa variable se asocie en mayor medida a ellos que a los maltratadores (Campbell et al., 2003; Echeburúa et al., 2008; Kivisto, 2015).

Recordando que, tal y como mencionan Bridger et al. (2017), los indicadores de suicidio reúnen las características necesarias para ser un predictor valioso del feminicidio (ya 
que, al no tener una prevalencia tan alta en el resto de la población general, se evitan altas tasas de falsos positivos). Las conclusiones del presente estudio pueden ayudar a la prevención de la violencia de género y de futuros feminicidios, a través de la actuación en ámbitos policiales, asistenciales y clínicos. En primer lugar, hay que concienciar sobre la importancia de que los distintos profesionales recojan la información sobre los indicadores de suicidio lo mejor posible, con la máxima atención, por su poder para ayudar a predecir el desenlace mortal si posteriormente se ponen en marcha mecanismos de seguimiento y de protección. En segundo lugar, los datos obtenidos invitan a ajustar los programas de intervención existentes con agresores, para que sean más eficaces y efectivos, destinando los recursos disponibles en la medida de lo posible a aquellos agresores que presenten indicadores de suicidio. También permiten ajustar los programas de intervención con víctimas, para que estas

sean conscientes del riesgo y sepan identificar señales de peligro en las amenazas o intentos de suicidio de su pareja.

Como limitación cabe reseñar que, en la muestra de feminicidas empleada para este estudio, los indicadores de suicidio se obtuvieron a posteriori del hecho en los casos sin denuncia previa, a partir de entrevistas con el entorno del autor y de la víctima. Esto pone en valor la importancia de analizar otras fuentes de información del entorno de la víctima cuando esta da el paso de denunciar a su agresor.

Por último, en cuanto a líneas de investigación futuras, estos resultados animan a buscar otros indicadores de riesgo de feminicidio en España que, de existir, podrían dar lugar a la elaboración y validación de un instrumento específico de valoración y predicción del riesgo de letalidad en los casos de violencia de género.

\section{REFERENCIAS}

Barber, C. W., Azrael, D., Hemenway, D., Olson, L. M., Nie, C., Schaechter, J., \& Walsh, S. (2008). Suicides and suicide attempts following homicide: victim-suspect relationship, weapon type, and presence of antidepressants. Homicide Studies, 12(3), 285-297

Barber, C. W., Azrael, D., Hemenway, D., Olson, L. M., Nie, C., Schaechter, J., \& Walsh, S. (2008). Suicides and suicide attempts following homicide: victim-suspect relationship, weapon type, and presence of antidepressants. Homicide Studies, 12(3), 285-297.

Belfrage, H., \& Rying, M. (2004). Characteristics of spousal homicide perpetrators: a study of all cases of spousal homicide in Sweden 1990-1999. Criminal Behaviour and Mental Health, 14(2), 121-133.

Bridger, E., Strang, H., Parkinson, J., \& Sherman, L. W. (2017). Intimate partner homicide in England and Wales 2011-2013: Pathways to prediction from multi-agency domestic homicide reviews. Cambridge Journal of Evidence-Based Policing, 1(2-3), 93-104.

Button, I. M., Angel, C., \& Sherman, L. W. (2017). Predicting domestic homicide and serious violence in Leicestershire with intelligence records of suicidal ideation or self-harm warnings: A Retrospective analysis. Cambridge Journal of Evidence-Based Policing, 1(2-3), 105-115.

Campbell, J. C. (2004). Danger assessment. Johns Hopkins University, School of Nursing. Recuperado de https://www.dangerassessment.org/DATools.aspx

Campbell, J. C., Glass, N., Sharps, P. W., Laughon, K., \& Bloom, T. (2007). Intimate partner homicide: review and implications of research and policy. Trauma, Violence, y Abuse, 8(3), 246-269.

Campbell, J. C., Webster, D., Koziol-McLain, J., Block, C., Campbell, D., Curry, M. A., ... \& Sharps, P. (2003). Risk factors for femicide in abusive relationships: Results from a multisite case control study. American Journal of Public Health, 93(7), 1089-1097.

Chalkley, R., \& Strang, H. (2017). Predicting domestic homicides and serious violence in Dorset: A replication of Thornton's Thames Valley analysis. Cambridge Journal of Evidence-Based Policing, 1(2-3), 8192.

Comstock, R. D., Mallonee, S., Kruger, E., Rayno, K., Vance, A., \& Jordan, F. (2005). Epidemiology of homicide-suicide events: Oklahoma, 1994-2001. The American journal of forensic medicine and pathology, 26(3), 229-235.

Corradi, C., \& Stöckl, H. (2014). Intimate partner homicide in 10 European countries: Statistical data and policy development in a cross-national perspective. European Journal of Criminology, 11(5), 601618.

De Juan, M., González, J. L., Liberatore, F., Quijano, L. \& Torrecilla, J. L. (2017). Robustez de los actuales formularios de valoración policial del riesgo de violencia de género (Informe Técnico del Instituto de Ciencias Forenses y de la Seguridad en colaboración con el Gabinete de Coordinación y Estudios de la Secretaría de Estado de Seguridad). Madrid.

Echeburúa, E., Fernández-Montalvo, J., \& Corral, P. D. (2008). ¿Hay diferencias entre la violencia grave y la violencia menos grave contra la pareja?: un análisis comparativo. International Journal of Clinical and Health Psychology, 8 (2).

Eke, A. W., Hilton, N. Z., Harris, G. T., Rice, M. E., \& Houghton, R. E. (2011). Intimate partner homicide: Risk assessment and prospects for prediction. Journal of Family Violence, 26(3), 211-216.

González, J. L., Garrido, M. J., López, J. J., Muñoz, J. M., Arribas, A., Carbajosa, P., \& Ballano, E. (2018). Revisión pormenorizada de homicidios de mujeres en las relaciones de pareja en España. Anuario de Psicología Jurídica, 28(1), 28-38.

González, J. L., López-Ossorio, J. J. \& Muñoz (2018). La valoración policial del riesgo de violencia contra la mujer pareja en España. Madrid: Ministerio del Interior.

González, J. L., Sánchez, F., López-Ossorio, J. J., Santos, J. \& Cereceda, J. (2018). Informe sobre el Homicidio en España. Madrid: Ministerio del Interior.

Kivisto, A. J. (2015). Male perpetrators of intimate partner homicide: A review and proposed typology. Journal of the American Academy of Psychiatry and the Law online, 43(3), 300-312. 
Kropp, P. R., \& Hart, S. D. (2000). The Spousal Assault Risk Assessment (SARA) guide: Reliability and validity in adult male offenders. Law and Human Behavior, 24(1), 101-118.

Liem, M., Hengeveld, M., \& Koenraadt, F. (2009). Domestic homicide followed by parasuicide: a comparison with homicide and parasuicide. International Journal of Offender Therapy and Comparative Criminology, 53(5), 497-516.

Loinaz, I., Marzabal, I. \& Andrés-Pueyo, A. (2018). Risk factors of female intimate partner and non-intimate partner homicides. European Journal of Psychology Applied to Legal Context, 10(2).

López-Ossorio, J. J., Carbajosa, P., Cerezo-Domínguez, A. I., GonzálezÁlvarez, J. L., Loinaz, I., \& Muñoz-Vicente, J. M. (2018). Taxonomía de los homicidios de mujeres en las relaciones de pareja. Psychosocial Intervention, 27(2), 95-104.

López-Ossorio, J. J., González, J. L., Buquerín, S., García, L. F., \& Buela-Casal, G. (2017). Risk factors related to intimate partner violence police recidivism in Spain. International Journal of Clinical and Health Psychology, 17(2), 107-119.

López-Ossorio, J. J., González-Álvarez, J. L., \& Andrés-Pueyo, A. (2016). Eficacia predictiva de la valoración policial del riesgo de la violencia de género. Psychosocial Intervention, 25(1), 1-7.

López-Ossorio, J.J., González-Álvarez, J.L., Muñoz-Vicente, J.M., Urruela, C. \& Andrés-Pueyo, A. (2019). Validation and Calibration of the Spanish Police Intimate Partner Violence Risk Assessment System (VioGén). Journal of Police and Criminal Psychology. DOI: 10.1007/s11896-019-09322-9

López-Ossorio, J.J., Loinaz, I. \& González-Álvarez, J.L. (2019). Protocolo para la valoración policial del riesgo de violencia de género (VPR4.0): revisión de su funcionamiento. Revista Española de Medicina Legal, 45(2), 52-58. DOI: 10.1016/j.reml.2019.01.002

Pardo, A., \& San Martín, R. (2015). Análisis de Datos en Ciencias Sociales y de la Salud II. Editorial Síntesis: Madrid.

Richards, L. (2009). 15 High Risk Factors of Serious Harm and Homicide in Domestic Abuse (DA) Cases. Recuperado de: https://www.dashriskchecklist.co.uk/wpcontent/uploads/2016/09 /One_Page_High_Risk_Factor_Definitons_for_Domestic_pdf

Thornton, S. (2017). Police Attempts to Predict Domestic Murder and Serious Assaults: Is Early Warning Possible Yet?. Cambridge Journal of Evidence-Based Policing, 1(2-3), 64-80.

Vatnar, S. K. B., Friestad, C., \& Bjørkly, S. (2019). The influence of substance use on intimate partner homicide: Evidence from a Norwegian national 22-year cohort. International Journal of Forensic Mental Health, 18(2), 99-110. 